\title{
Can't get no diffraction?
}

In the year 1900, no one would have guessed that light - to all appearances an ethereal wave-like phenomenon - might take the form of localized particles. Nor would anyone have expected that the billiardball atoms of physical matter would turn out to act as waves, showing diffraction and interference much like light. In that pre-quantum age, light and matter sat on two sides of a conceptual divide.

That divide was soon annihilated, of course, when Einstein, in 1905, showed light to be absorbed as discrete photons, and Davisson and Germer, in 1927, demonstrated that slow-moving electrons create a diffraction pattern when reflected from a crystal surface. This effect had been foreseen by Louis de Broglie, whose $\mathrm{PhD}$ thesis of 1924 was the first attempt to obliterate the wave-particle divide: "When I conceived the first basic ideas of wave mechanics," he later wrote, "I was guided by the aim to perform a real physical synthesis, valid for all particles, of the coexistence of the wave and of the corpuscular aspects."

Present-day quantum theory is the long outgrowth of de Broglie's idea, but of course also still subject to revision. Ironically, some of the strongest tests of quantum theory at present originate in diffraction experiments directly analogous to those of Davisson and Germer, yet also different in a way that de Broglie may not have imagined - with light itself forming the physical structure that plays the role of the crystal, and material particles, even large complex molecules, the things being diffracted.

A coherent beam of particles of any kind can show wave-like diffraction effects when made to pass through a grating of slits. Far away from the grating, the density of detected particles should show an oscillatory pattern with well-defined maxima and minima along different directions. However, the ease of seeing such phenomena decreases with increasing particle mass and velocity.

That's because the de Broglie wavelength, $\lambda_{\mathrm{DB}}$, associated with a particle is inversely proportional to its momentum, and pronounced diffraction effects only occur if the spacing of the grating is small enough to be comparable to the incident wavelength. For low-energy electrons, the periodic arrangement of atoms in a crystal provides a convenient grating of the right size. Observing the same kind of thing for larger particles - a basic test of the validity of quantum principles - requires gratings with finer resolution.

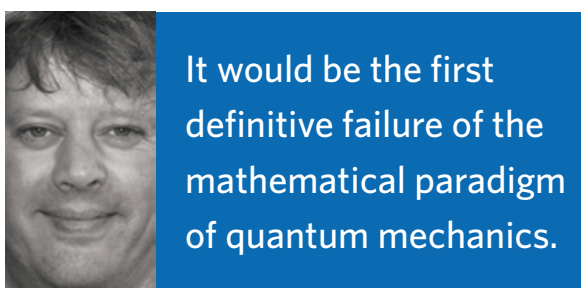

Experiments of this kind over the past two decades have demonstrated diffraction phenomena for a host of atoms including hydrogen, helium, sodium and even complex fullerene molecules such as $\mathrm{C}_{60}$. In 1999, for example, physicists in Vienna sent a beam of $\mathrm{C}_{60}$ molecules with a velocity of about $100 \mathrm{~ms}^{-1}$ onto a grating with a slit separation of $100 \mathrm{~nm}$ and detected interference maxima and minima at a distance of about $1 \mathrm{~m}$ (M. Arndt et al. Nature 401, 680-682; 1999).

Seeing similar effects for even larger particles seems to require a shift in strategy. Most diffraction experiments have explored so-called far-field diffraction - that is, the effects apparent when viewed sufficiently far from the source, where the wave fronts are effectively plane waves. Achieving observable diffraction in this setting requires molecular beams collimated more precisely than the diffraction angle. In the experiments with $\mathrm{C}_{60}$, for example, the beam had to be collimated to less than $10 \mu \mathrm{m}$, which also strongly reduced the detected particle flux. This technique runs into several practical limitations if applied to even larger particles for which the de Broglie wavelengths tend to be smaller still.

A promising alternative is to turn away from the far field, and instead detect diffraction effects in the near field, closer to the grating (K. Hornberger et al. Rev. Mod. Phys.; in the press). The physics is more difficult to analyse, but the near field offers advantages - in particular, the required spacing of the grating scales inversely to the square root of the particle mass, rather than simply in inverse proportion to the mass. Moreover, the technique does not require molecular beams of very narrow width.

Mathematically, when a plane wave illuminates a grating of spacing $d$, the intensity pattern across the grating will be reproduced exactly at the so-called Talbot length $L_{\mathrm{T}}$ in the forward direction, where $L_{\mathrm{T}}=d^{2} / \lambda_{\mathrm{DB}}$. This intensity distribution is an inherent reflection of wave physics, and it's detection - by observing the distribution of particles on some clean surface, for example - demonstrates diffraction. This technique was demonstrated over a decade ago in experiments.

But everything can be taken a step further by replacing the physical grating with a grating made entirely of light. As it turns out, the physical grating introduces limitations traced to van-der-Waals-type weak interactions between the particles and the walls of the grating. These can be eliminated by replacing the grating with a standing-wave laser field of the same periodicity. Using this technique, Hornberger and colleagues have recently demonstrated clear quantum diffraction for a variety of organic molecules, some containing more than 400 atoms and weighing as much as 7,000 amu (S. Gerlich et al. Nature Commun.

\section{2, 263; 2011).}

The pay-off of this experimental advance is the chance to test various theories proposed to reconcile fundamental puzzles in quantum physics. Why, for example, do small quantum particles such as electrons routinely exist in superpositions - and therefore show interference effects whereas we never see such effects for large objects on the macroscopic scale? One proposal, embodied in so-called continuous spontaneous localization theories, is that the linear Schrödinger equation is actually incomplete, and needs to be generalized to include real nonlinear processes by which the wavefunction occasionally collapses from superposed states into states with precise spatial positions.

The parameters of these hypothetical theories can be chosen so that tiny particles such as electrons act just as standard quantum theory says they should, while superpositions in large ensembles (of order $10^{24}$ particles) collapse very rapidly. This would explain the quantum-classical divide quite naturally. But it implies that experiments aiming to see diffraction in sufficiently large particles should begin to fail.

As Hornberger et al. point out, the experiments are getting close to the point where they will begin to constrain the parameters in these theories - if they continue to see diffraction. If they do not, it would be the first definitive failure of the standard mathematical paradigm of quantum mechanics, and could help chart the way to a better theory beyond.

MARK BUCHANAN 